\title{
SAND99-3136C
}

\section{On the Construction of a Domain Language for a Class of Reactive Systems*}

\author{
Deepak Kapur ${ }^{\dagger}$ \\ University of New Mexico \\ .kapur@unm.edu
}

\section{Abstract}

A key step in the construction of high consequence software is its specification in a formal framework. In order to minimize the difficulty and potential for error, a specification should be expressed in a domain language supporting operators and structures that are intrinsic to the class of algorithms one wishes to specify.

In this paper we describe a language that is suitable for the algorithmic specification of software controllers for a class of reactive systems of which the Bay Area Rapit Transit (BART) system is an instance. We then specify an abstract controller for a subset of $B A R T$ using this language.

\section{Overview}

This paper describes our preliminary results in the development of a domain language and methodology suitable for the formal specification, design, and development of high integrity software-based controllers for train systems or systems having similar characteristics. The Bay Area Rapid Transit (BART) system is a prime example of the type of system in which we are interested. A document describing BART as well as what constraints its controller must satisfy can be found in [7].

The paper is organized as follows: Section 2 describes the issues surrounding the design of reactive systems and how such systems can be modeled. Section 3 discusses the predictive models and calculations which need to be performed by controllers of the sub-

\footnotetext{
*This work was supported by the United States Department of Energy under Contract DE-AC04-94AL85000. Sandia is a multiprogram laboratory operated by Sandia Corporation, a Lockheed Martin Company, for the United States Department of Energy.

†Deepak Kapur was also partially supported by NSF grant nos. CCR-9996150 and CDA-9503064.
}

\author{
Victor L. Winter \\ Sandia National Laboratories \\ vlwinte@sandia.gov
}

set of reactive systems on which we are focusing. Sections 4 and 5 introduce the notion of a profile and how it can be used to describe system behaviors as well as system constraints. Section 6 defines a domain language in which the calculations described in the previous section can be concisely specified. This domain language is designed with the knowledge that automated transformation will be used as a technique for deriving executable implementations from specifications. Section 7 gives a formal algorithmic specification for a train controller. The paper concludes with a discussion of conclusions and future work.

\section{A Model of Reactive Systems}

Abstractly speaking, a system design consists of various components and is intended to function in the presence of a specific set of environmental conditions. The components of the system as well as environmental elements may have characteristics that cannot be readily captured by predictive models. For example, in a robotic system, the time it takes for a conveyor belt to move an object from point $A$ to point $B$ might vary as a result of various factors that cannot be accurately modeled (e.g., fluctuations in the power supply, temperature, etc.). Similarly, weather conditions might effect the speed and acceleration of a train.

These examples serve to highlight that models are approximations of aspects of reality in which we are interested. In this paper, we use the term nondeterminism to refer to the difference between a model of a thing and the thing itself. The justification for the use of this term is that from the perspective of the model, these differences appear nondeterministic.

Depending on the system design, some kinds of nondeterminism may be safely ignored. For example, if the time it takes for a conveyor belt to move an object from point $A$ to point $B$ is unimportant with respect to a given margin of error (e.g., within a few sec- 


\section{DISCLAIMER}

This report was prepared as an account of work sponsored by an agency of the United States Government. Neither the United States Government nor any agency thereof, nor any of their employees, make any warranty, express or implied, or assumes any legal liability or responsibility for the accuracy, completeness, or usefulness of any information, apparatus, product, or process disclosed, or represents that its use would not infringe privately owned rights. Reference herein to any specific commercial product, process, or service by trade name, trademark, manufacturer, or otherwise does not necessarily constitute or imply its endorsement, recommendation, or favoring by the United States Government or any agency thereof. The views and opinions of authors expressed herein do not necessarily state or reflect those of the United States Government or any agency thereof. 


\section{DISCLAIMER}

\section{Portions of this document may be illegible in electronic image products. Images are produced from the best available original document.}


onds), then the effects of nondeterminism can be safely ignored. However, in many cases, the effects nondeterminism is consequential and cannot be ignored. It is the presence of this second type of nondeterminism that does not admit the possibility of precomputing a system behavior based solely on predictive models.

Systems in which consequential nondeterminisim is present must realize their behavior in a reactive manner. Sensors are added to a system design in order to overcome nondeterminism, and control elements are added in order to allow the behavior of various components of the system to be altered as result of the information obtained from the sensors, enabling a controller to compensate for the difference between the system and its model. We call systems having these design characteristics reactive systems. This definition is consistent with [5] in which a reactive system is defined as "a system that maintains an ongoing interaction with its environment, as opposed to computing some final value on termination".

The state of an ideal reactive system is described by two vectors. The first vector, $\vec{m}$, represents the values of the sensors in the system, and is called the vector of monitored variables. The second vector, $\vec{c}$, is called the vector of control.variables and represents those aspects of the system behavior that can be dynamically controlled.

- Let $\vec{m}=\left\{m_{1} \in D_{1}, \ldots, m_{j} \in D_{j}\right\}$ denote the observable state of the system.

- Let $\vec{c}=\left\{c_{1} \in C_{1}, \ldots, c_{k} \in C_{k}\right\}$ denote the vector of control variables of the system.

- Then $\vec{s}=\vec{m} \circ \vec{c}=\left\{m_{1}, \ldots, m_{j}, c_{1}, \ldots, c_{k}\right\}$ is the state of the system. Note that $\vec{m} \circ \vec{c}$ contains $\left|D_{1}\right| \times \ldots \times\left|D_{j}\right| \times\left|C_{1}\right| \times \ldots \times\left|C_{k}\right|$ states.

Henceforth, we will drop the vector notation and use $m, c$, and $s$ to denote the vectors describing the observable state space of a system, the vector of control variables, and the system respectively.

Informally stated, $m$ serves as a calibration point with respect to physical reality. That is, $m$ measures the observed state of the physical system and the information it contains can be used to update the state of our predictive system model. If this kind of update were not to take place, then nondeterministic factors could have a cumulative effect on the system model. What this means is that, over time, the state of the system model could diverge significantly from the state of the physical system. The role of $m$ is to recalibrate the system model in order to prevent this cumulative divergence from happening. The frequency of this recalibration depends on the predictive abilities of our models as well as the sensitivity of the system to sense and alter its behavior.

It should be noted that $m$ is also under the influence of nondeterminism. However, it is important to realize that the influence of nondeterminism on $m$ is not cumulative in nature and thus can be bounded in some manner (e.g., statistically in terms of a binomial distribution).

Given a recalibration interval of $\Delta t$, a reactive system controller performs the following sequence of steps:

1. Sense

- Determine the state of the system by obtaining $m$ and $c$.

2. React

- Use the state of the system together with predictive models of the system behavior to compute the action to be taken. This action is expressed in the form of a control vector c.

- Output $c$.

\section{Goto step 1.}

Let $m_{t_{i}}$ denote the observed state at time $t_{i}$, and let $t_{i}+\gamma t$ denote the time at which the control vector, $c_{t_{i}+\gamma t}$, is sent/output to the system. It should be noted that in general, the state of the system at time $t_{i}+\gamma t$ will be different from the state at time $t_{i}$. Depending on the resolution/sensitivity of the system sensors, this difference may or may not be observable through $m$. Either way, the reality is that our react computation is based on $m_{t_{i}}$, but the reaction is performed by the system when it is in state $m_{t_{i}+\gamma t}$. 


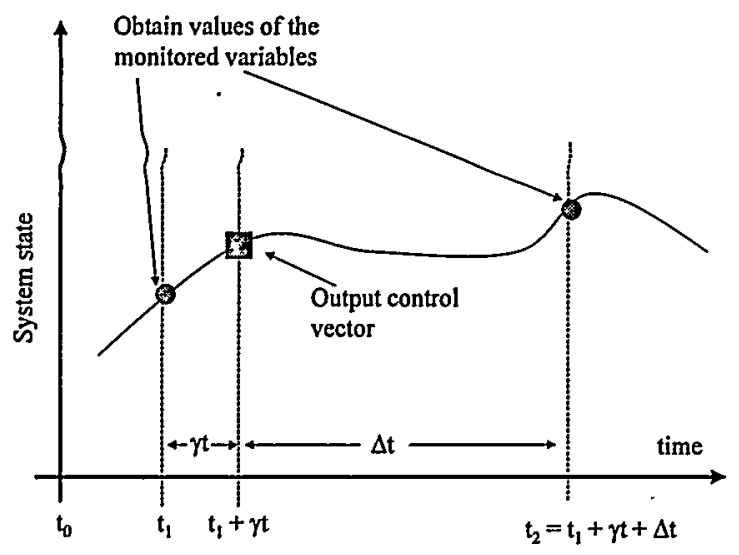

Figure 1: The Control of a Reactive System

The discrepancy between sense time and react time can be addressed in two ways:

1. Given the time, $\gamma t$, needed to compute the react step, a predictive model of the system can be used to compute $m_{t_{i}+\gamma t}$, and the react computation can be performed with respect to $m_{t_{i}+\gamma t}$ rather than $m_{t_{i}}$. The rational behind this approach is based on the assumption that given $m_{t_{i}}$, our ability to predict the system state at time $t_{i}+\gamma t$ is more accurate than simply using $m_{t_{i}}$.

2. One can assume that the system is continuous over small time intervals and that $\gamma t$ qualifies as a small time interval. The idea here is that whatever control vector, $c_{t_{i}}$, computed for $m_{t_{i}}$ would also be acceptable for $m_{t_{i}+\gamma t}$. In this approach, we are for all practical purposes assuming that $\gamma t=0$.

While approach 1 given above can be theoretically interesting, in practice it is often times not feasible when $\gamma t$ is very small. Essentially what happens as $\gamma t$ becomes small is that a point is reached where the change in system state cannot be observed through $m$ because it exceeds the resolution of the sensors. Thus the sensors see $m_{t_{i}}=m_{t_{i}+\gamma t}$. For this and other similar reasons (e.g., the resolution of the control variables), when $\gamma t$ is sufficiently small, the difference between $m_{t_{i}}$ and $m_{t_{i}+\gamma t}$ essentially gets "lost in the noise", which has the end effect of setting $\gamma t=0$.

In our approach we make the assumption that $\gamma t<<\Delta t$ and in our system model we set $\gamma t=0$. It should be noted that making this assumption is not particularly dangerous because, given an implementation one can always measure $\gamma t$ to determine if this assumption is reasonable.
When making the assumption that $\gamma t=0$, it becomes notationally convenient to be able to distinguish the old value of $c_{t_{i}}$ that resulted from the previous sense-react step with the newly computed value of $c_{t_{i}}$. We make this distinction by superscripting the time index with a "-" and a " + " respectively. In the limit, as $\gamma t \rightarrow 0, t_{i}^{-} \rightarrow t_{i}$. and $t_{i}^{+} \rightarrow t_{i}$

By letting $\gamma t=0$, a reactive system controller performs the following steps:

1. Let $t_{i}^{-}$denote the current time.

2. $\gamma t$ Step:

(a) At time $t_{i}^{-}$read the values of the sensor variables and compute the observable state vector, $m_{t_{\bar{i}}^{-}}$, of sensor variables. The system state at this point is $s_{t_{i}^{-}}=\left(m_{t_{i}^{-}}^{\prime}, c_{t_{i}^{-}}^{\prime}\right)=$ $\left(m^{\prime}, c^{\prime}\right)_{t_{i}^{-}}$

(b) Compute the control variables, $c_{t_{i}^{+}}$, corresponding to $s_{t_{\bar{i}}}$

(c) At time $t_{i}^{+}$output $c_{t_{i}^{+}}$to the system. The system state is now: $s_{t_{i}^{+}}=\left(m_{t_{\bar{i}}^{-}}^{\prime}, c_{t_{i}^{+}}\right)=$ $\left(m^{\prime}, c\right)_{t_{i}^{+}}$

3. $\Delta t$ Step:

(a) Let the system run for a small time interval $\Delta t$. The system state is now: $s_{t_{i}+\Delta t}=$ $\left(m_{t_{i}+\Delta t}, c_{t_{i}^{+}}\right)=(m, c)_{t_{i}+\Delta t}$

4. Go to step 1 .

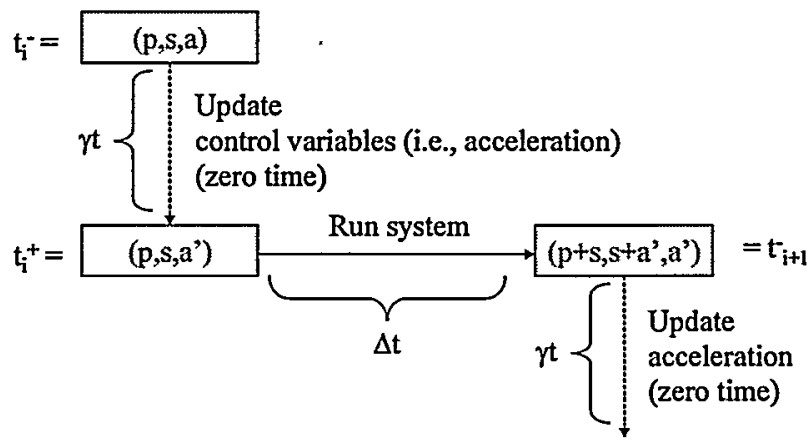

Figure 2: Model of Time 
Note that in the figure above, $s$ and $a$ are normalized with respect to the time unit $\Delta t$. Symbolically the transition sequence of the system is as follows:

$$
\left(m_{1}, c_{1}\right)_{t_{i}^{-}} \stackrel{\gamma t=0}{\longrightarrow}\left(m_{1}, c_{2}\right)_{t_{i}^{+}} \stackrel{\Delta t}{\longrightarrow}\left(m_{2}, c_{2}\right)_{t_{i}+\Delta t} \stackrel{\gamma t=0}{\longrightarrow} \ldots
$$

\section{Domain Specific Predictive Models}

In the previous section, we described how general reactive systems can be modeled. In this section we focus our attention on the types of computations that are needed in order to safely control the behavior of a reactive system such as BART. The subset of BART that we will primarily consider consists of two trains, an object train which is behind a lead train, and a track. We are aware that our model omits signals and stations, and will address how they can be modeled later in this paper.

We assume that sensors exist that monitor the position and speed of each train, and control variables exist for changing the acceleration of each train. The track consists of track segments, each of which can safely support a predetermined maximum train speed. Given this system definition, we say the lead train is constrained by the speed limit of the track segment on which it is currently traveling, and the object train is constrained by both the lead train and its corresponding track segment. A train violates the track segment constraint if it exceeds the speed limit of the track segment on which it is traveling. Similarly, the object train violates the lead train constraint if it gets so close to the lead train that a collision could occur (we will elaborate on this shortly). Later in this paper we discuss how a specification for this simple system can be easily extended to handle additional constraints such as track signals and stations.

As a starting point we assume that the state space of BART is modeled in the manner described in Section 2. Given such a system model we now focus on how transitions, which result from acceleration commands, can be calculated in a predictive manner. $\mathrm{Ab}$ stractly speaking, the predictive calculation of these transitions allows the predictive exploration of transition sequences which is essential to enable desired behaviors to be realized.

With the exception of the first train, the train control problem for BART is generic, in the sense that every train can be viewed as being an object train with respect to some lead train. This allows us to focus our attention on the specification and implementation of a controller for an object train with negligible loss of generality.

Our objective is to specify an abstract algorithm, called a controller, that when given a system state, $s_{t_{i}}$, computes an acceleration, $a_{t_{i}}$, of an object train in such a manner that the object train never violates either the lead train constraint or the track speed constraint. A more complex system is then just a collection of such controllers where each train is viewed as an object train by its corresponding controller.

Trains in BART have certain physical characteristics that, if not modeled carefully, can present substantial computational challenges to the construction of efficient controllers. These characteristics are: (1) a train (e.g., the object train) can take a long time to stop-even when the emergency brake is applied, (2) a train cannot instantaneously switch from positive to negative acceleration, and (3) a train cannot instantaneously go from negative acceleration to full negative acceleration. Because of these physical limitations in reducing speed, the object train controller must anticipate potential problems (e.g., exceeding an upcoming track speed limit, or colliding with the leading train) well in advance and react accordingly. In order to be able to react "in advance", the controller must have access to a model capable of predicting the trains behavior. Note that such a behavior corresponds to a transition sequence in a model of the train. A key problem that this model must be able to solve is: "Given that a train is has a speed $s_{1}$ at position $p_{1}$, find the set of accelerations which do not exclude the possibility for the train to have a speed $s_{2}$ at position $p_{2}$." Given the ability to solve this type of model, a controller can search through this set of possible acceleration values for a value that satisfies some additional property (e.g., optimality, or smoothness).

\subsection{Partitioning of the State Space}

Reacting to a constraint, like a track speed limit, that is constant over time is conceptually straightforward. However, reacting to the behavior of a leading train is somewhat more problematic. The reason for this is that some assumptions should be made regarding the future behavior of a lead train (e.g., a controller becomes very inefficient if it always assumes that the lead train is at a dead stop). Nevertheless, not many assumptions can be made about the future behavior of a leading train. For example, one cannot unilaterally conclude that just because the leading train is traveling $50 \mathrm{mph}$ at time, $t_{i}$, that it will be traveling in the neighborhood of $50 \mathrm{mph}$ at time $t_{i}+\Delta t$. The reason for this being that the lead train may derail in which 
case, it is conservatively assumed to instantaneously come to a dead stop. Formally stated, the state of a train is defined by a triple $(p, s, a)$ denoting it's position, speed, and acceleration. A derailment then is modeled as the transition: $(p, s, a) \Rightarrow(p, 0$, derail $)$. Also without global information, one cannot make any assumptions whether the control function for the lead train would want to increase or decrease the lead train's speed during the next time step.

A conceptually clean way of delineating the assumptions that can be made about the behavior of the lead train is to partition the state space, $\mathcal{S}$, of the system into normal and abnormal subsets, denoted by $\mathcal{N} \_$S and $\mathcal{A} B \_S$ respectively.

- $\mathcal{S}=\mathcal{N}_{-} \mathcal{S} \cup \mathcal{A B} \_S$

- $\mathcal{N} \mathcal{S} \cap \mathcal{A} \mathcal{B}_{-} \mathcal{S}=\emptyset$

Similarly the set of acceleration values can also be partitioned into normal, $\mathcal{N}_{\mathcal{A}} \mathcal{A}$, and abnormal, $\mathcal{A} \mathcal{B}_{\mathcal{A}} \mathcal{A}$, subsets. However, in BART, the set of normal accelerations that a train can realize (i.e., that it can transition to) is a function of the current acceleration. For this reason, it makes sense to view $\mathcal{N}_{-} \mathcal{A}$ as a function from normal accelerations to normal acceleration sets, rather than as a fixed partition.

- $\mathcal{A}\left(a_{i}\right)=\mathcal{N}_{\mathcal{A}} \mathcal{A}\left(a_{i}\right) \cup \mathcal{A} \mathcal{B}_{\mathcal{A}} \mathcal{A}$

- $\mathcal{N}_{\mathcal{A}} \mathcal{A}\left(a_{i}\right) \cap \mathcal{A B}_{\mathcal{A}} \mathcal{A}=\emptyset$

In this approach, given an acceleration, $a_{1}$, the set of transitions belonging to $\mathcal{N}_{-} \mathcal{A}\left(a_{1}\right)$ includes the accelerations that can be realized by a controller with the exception of the emergency stop command. In contrast, $\mathcal{A} \mathcal{B}_{-} \mathcal{A}$ is a fixed partition that includes drastic acceleration changes that can be realized by the environment, and are, hence, physically possible, even though they cannot be realized by a controller. Thus derail $\in \mathcal{A B} \_\mathcal{A}$.

The emergency stop command should never be issued under normal conditions. It is a possible object train's response to a drastic need to stop (e.g., the derailment of its lead train). Because of this, we place the emergency stop command in $\mathcal{A} \mathcal{B}_{-} \mathcal{A}$, making $\mathcal{A} \mathcal{B}_{\mathcal{A}} \mathcal{A}=\{$ derail, emergency_stop $\}$.

\subsubsection{Basic Safety}

In this section, we define safety from the perspective of the object train, in its most basic terms. Namely, the system is in an unsafe state if a collision between the lead train and the object train has taken place, or if the object train violates the speed limit of the track. We define states that are unsafe (from the perspective of the object train) as being abnormal. We also define as abnormal, states in which the object train is commanding an emergency_stop. From these two base cases, we then define as abnormal, the set of all states in which control of the object train alone is not suffcient to prevent an abnormal (base) state from being reached.

After discussing basic safety in depth we then introduce and discuss, in Section 5.1, a refined notion of safety where the system is in an unsafe state if the object train is so close to the lead train that an emergency stop command, given under a postulated set of worst case conditions, is not sufficient to prevent a collision from occurring. We then briefly discuss how, when given a function for computing the worst case stopping distance, our model can be easily extended so that this distance is accounted for.

Given a two train system whose state is modeled by the vector $s$, we introduce a predicate hazard_object_train which is informally defined as follows:

\section{Definition 1 hazard_object_train(s) = true iff}

1. The position of the object train is greater than or equal to the position of the lead train (i.e., a collision has occurred), or

2. The speed of the object train exceeds the speed limit of the track segment on which it is currently traveling.

Abstractly, the two conditions stated above define a set of states that must always be avoided by the object train. This set forms the basis for defining the set of "abnormal" system states, which may not be directly hazardous, but which form part of sequences leading to hazardous states in a manner that is beyond the control of the normal operation of the object train. In other words, the object train can be forced into a hazardous state.

It is worth mentioning that addition states, corresponding to various system requirements, can be easily added to the set of hazardous states. For example, the requirement that an object train stop at signals can be easily defined.

Definition 2 From the perspective of the object train, the abnormal subset, $\mathcal{A B}_{-} \mathcal{S}$, of the system state space is defined as follows: 


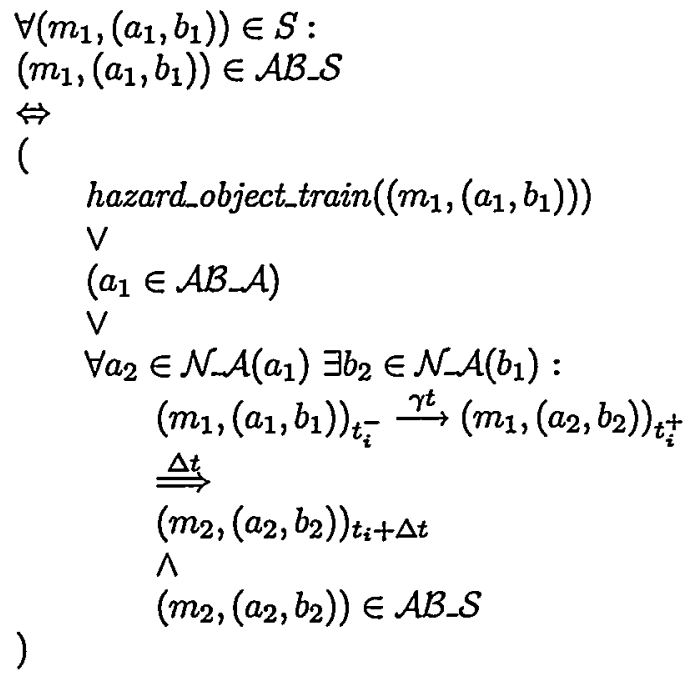

The definition of a normal state is then the negation of the above definition. Note that the negation of the last part of the above expression begins $\exists a_{2} \in \mathcal{N}_{-} \mathcal{A}\left(a_{1}\right) \forall b_{2} \in \mathcal{N}_{-} \mathcal{A}\left(b_{1}\right)$. This statement is a very strong statement, which is much stronger than $\forall b_{2} \in \mathcal{N}_{-} \mathcal{A}\left(b_{1}\right) \exists a_{2} \in \mathcal{N}_{-} \mathcal{A}\left(a_{1}\right)$. However, this strength is precisely needed for BART because it reflects our assumptions regarding future accelerations of the lead train. In particular, according to our assumptions, the controller for the object train does not have knowledge of future acceleration commands for the lead train. Note that given a different set of assumptions regarding the future behavior of the lead train, the expressions defining normal and abnormal states may be weakened or strengthened accordingly.

We now define safety in the context of the above definition of $A B_{-} S_{\text {. }}$. Given that the system is in a normal state to begin with and that the acceleration computed for the lead train is normal, the goal of the object train controller, from the standpoint of safety, is to compute an object train acceleration such that the resulting state is not an element of $\mathcal{A B} S$.

Furthermore, if the acceleration realized by the lead train is abnormal, then the object train can go into emergency stop, if needed. Formally this is stated as follows:

- Let $\left(m_{1},\left(a_{1}, b_{1}\right)\right)_{t_{i}^{-}}$denote the current system state.

- Let $f: \mathcal{S} \rightarrow \mathcal{A}$ denote a controller function for the object train.

- Let $\left(m_{2},\left(a_{2}, b_{2}\right)\right)$ denote the state resulting from:

$$
\left(m_{1},\left(a_{1}, b_{1}\right)\right)_{t_{\bar{i}}^{-}}
$$

$$
\begin{aligned}
& \left(m_{1},\left(a_{2}, b_{2}\right)\right)_{t_{i}^{+}} \\
& \stackrel{\Delta t}{\Longrightarrow} \\
& \left(m_{2},\left(a_{2}, b_{2}\right)\right)_{t_{i}+\Delta t}
\end{aligned}
$$

Definition 3 safe $(f) \Leftrightarrow$

$$
\begin{aligned}
& \text { ( } \\
& \forall\left(m_{1},\left(a_{1}, b_{1}\right)\right) \in \mathcal{N}_{-} \mathcal{S} \\
& \forall b_{2}:\left(b_{1} \in \mathcal{A} \mathcal{B}_{-} \mathcal{A} \vee b_{2} \in \mathcal{N} \mathcal{A}\left(b_{1}\right)\right) \\
& \overrightarrow{(} \\
& \left(f\left(\left(m_{1},\left(a_{1}, b_{1}\right)\right)\right) \in \mathcal{N} \_\mathcal{A}\left(a_{1}\right)\right) \\
& \wedge \\
& )^{\left.\left(m_{2},\left(f\left(\left(m_{1},\left(a_{1}, b_{1}\right)\right)\right), b_{2}\right)\right) \in \mathcal{N}_{-} \mathcal{S}\right)} \\
& )
\end{aligned}
$$

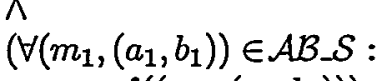

$$
\begin{aligned}
& \left.f\left(\left(m_{1},\left(a_{1}, b_{1}\right)\right)\right)=\text { emergency_stop }\right)
\end{aligned}
$$

From a safety perspective, the challenge at this point is to design and correctly implement a controller function $f$ that correctly realizes the definition of safety given above, and performs this computation in a relatively efficient manner. In the following sections, we introduce the notion of a profile and describe how profiles can be used to specify the constraints of a system and how they can be used to effectively compute accelerations that are safe.

\section{Discrete Profiles}

Virtually all aspects of the BART system can be modeled and reasoned about from the perspective of position and speed. As such, a position-speed tuple is a fundamental information unit with respect to formalizing and understanding the system. A discrete profile is the aggregation of position-speed tuples into a sequence.

A discrete profile is a sequence of positionspeed tuples arranged in a strict monotonically ascending order according to position. Let $P=$ $\left.<\left(p_{1}, s_{1}\right), \ldots,\left(p_{n}, s_{n}\right)\right\rangle$ denote an arbitrary discrete profile. Then $\forall i, j:\left(p_{i}, s_{i}\right) \in P \wedge\left(p_{j}, s_{j}\right) \in P \wedge i<$ $j \rightarrow p_{i}<p_{j}$.

Profiles (discrete and continuous-discussed in Section 4.2) provide an elegant and uniform way of formally modeling elements of the system such as the (1) track speed limits, (2) signals, and (3) stations. Furthermore, constraints of the system such as (1) avoid 
getting too close to the lead train, (2) avoid exceeding the speed limit of the track, (3) avoid crossing a signal that is in "stop" mode, and (4) stop at all stations, can all be expressed as properties of profiles.

\subsection{Describing BART in Terms of Pro- files}

We describe the elements of the BART system in terms of profiles as follows:

- track - the track can be described as a profile of position-speed tuples, $\left(p_{i}, s_{i}\right)$, where $s_{i}$ is the maximum speed at position $p_{i}$.

- signals - Let $\left\langle p_{1}, \ldots, p_{j}\right\rangle$ denote a sequence of monotonically increasing positions corresponding to the location of signals on the track. Let $\max$ denote the maximum speed limit possible, and let $\Delta d$ denote a small distance (e.g., one foot). Let the tuple $\left\langle\left(p_{k}, 0\right),\left(p_{k}+\Delta d, \max \right)\right\rangle$ denote "stop" for the signal at position $p_{k}$ and let the tuple $<\left(p_{k}, \max \right),\left(p_{k}+\Delta d, \max \right)>$ denote "go". By combining this description of each signal into a profile, the collective state of the signals can be expressed with respect to a given time $t$.

- stations - Stations are essentially modeled in the same manner as signals. We assume a station controller function similar to a signal controller function in the sense that it generates profiles that (1) force trains to stop at the required stations (e.g., by setting the speed at the station position to zero), and (2) releases them (e.g., by setting the speed at the station position to $\max$ ) after some suitable waiting period. The stations profile can be controlled separately (i.e., externally) from the controller or can be controlled within the object train controller itself.

\subsubsection{Stopping Profiles}

A profile of particular interest is the stopping profile of a train. Given a system state $\left(m_{1},\left(a_{1}, b_{1}\right)\right)_{t_{i}^{-}}$, the stopping profile for the object train is obtained by reducing the acceleration of the object train as much as is allowed under normal conditions, until the speed of the object train is $0 \mathrm{mph}$. A similar procedure is followed in order to obtain the stopping profile for the lead train.

When talking about and calculating stopping profiles, it becomes useful to separate from $s$, those variables specifically describing the train for which the stopping profile is to be computed. Let the variable lead_train denote the vector of monitored and controlled variables that describe the state of the lead train. In our model, the lead_train vector consists of two monitored variables describing the position and speed of the lead train, and one control variable describing its state of acceleration. A similar vector exists for the object train.

A stopping profile is defined as follows:

- Let $\left(p_{1}, s_{1}, a_{1}\right)_{t_{i}^{-}}$denote the vector describing the initial state of a train just prior to the react step.

- Let neg: $\mathcal{A} \rightarrow \mathcal{A}$ denote a function from normal accelerations to normal accelerations. This function takes a normal acceleration as input and returns the minimum normal acceleration (i.e., it tries to brake as quickly as possible under normal conditions).

- The stopping profile, $<\left(\dot{p}_{1}, s_{1}\right), \ldots,\left(p_{n}, s_{n}\right)>$, is obtained by extracting the position speed tuples from the corresponding sequence:

$$
\begin{aligned}
& \left(p_{1}, s_{1}, a_{1}\right)_{t_{i}^{-}} \stackrel{t_{c}=0}{\longrightarrow}\left(p_{1}, s_{1}, n e g\left(a_{1}\right)\right)_{t_{i}^{+}} \\
& \stackrel{\Delta t}{\Longrightarrow} \\
& \left(p_{2}, s_{2}, n e g\left(a_{1}\right)\right)_{t_{i}+\Delta t} \stackrel{t_{c}=0}{\longrightarrow} \ldots \\
& \stackrel{\Delta t}{\Longrightarrow} \\
& \left(p_{n}, 0, n^{\prime} g^{n}\left(a_{1}\right)\right)_{t_{i}+n * \Delta t}
\end{aligned}
$$

An important point concerning stopping profiles is that if the stopping profile of the lead and object trains are computed with respect to the same system state, then in effect, we have an implicit universal cock. For example, let $\left(p_{1}, s_{1}, a_{1}, q_{1}, t_{1}, b_{1}, \ldots\right)$ denote a system state where $\left(p_{1}, s_{1}, a_{1}\right)$ and $\left(q_{1}, t_{1}, b_{1}\right)$ denotes the position, speed, and acceleration of the object train and lead train respectively. In this context, let $\left\langle\left(p_{1}, s_{1}\right), \ldots,\left(p_{n}, s_{n}\right)\right\rangle$ and $\left\langle\left(q_{1}, t_{1}\right), \ldots,\left(q_{n}, t_{n}\right)\right\rangle$ denote the stopping profiles of the object train and lead train. Since each point corresponds to a clock tick, and since $\left(p_{1}, s_{1}\right)$ and $\left(q_{1}, t_{1}\right)$ correspond to the same clock tick, say time $e_{1}$, we have $\left(p_{i}, s_{i}\right)$ and $\left(q_{i}, t_{i}\right)$ occurring at time . $_{\text {. }}$

This notion of a universal clock, with respect to stopping profiles, is important to intuitively understanding why our definitions and theorems do not explicitly refer to time.

\subsection{Discrete and Continuous Profiles}

Our experiences in modeling BART leads us to believe that behavior in these kinds of reactive systems 
is most directly expressed and understood in a continuous framework. For example, whether the movement of a train over time satisfies or violates particular constraint. When thinking in terms of discrete representations, it becomes very easy to make numerous errors. Generically speaking, these errors would best be described as "off by one" errors. However, in spite of this difficulty in dealing with discrete profiles, profiles are ultimately expressed in their discrete form. This is due to practical considerations and limitations. For example, in BART, the track is described as a discrete profile. Nevertheless, it should be noted that even though the track is described in terms of a discrete profile it has a continuous semantics.

Let track_profile $=\left\langle\left(p_{1}, s_{1}\right), \ldots,\left(p_{n}, s_{n}\right)\right\rangle$. be the discrete profile describing the track. The purpose of this profile is to describes the maximum speed limit that is allowed at each position of the track. The algorithm for determining the speed limit for a position, $p_{k}$, that is not an element of a tuple in track_profile is as follows:

- Find adjacent tuples $\left(p_{i}, s_{i}\right)$ and $\left(p_{i+1}, s_{i+1}\right)$ in track_profile such that $p_{i}<p_{k}<p_{i+1}$

- The speed limit at position $p_{k}$ then is $s_{i}$

Using this paradigm we can extend a discrete profile to a continuous profile in the following manner:

Let $d p$ denote a discrete profile whose ordering of tuples is strictly monotonic on $p$ with respect to the relation $<$.

$$
\begin{aligned}
& \text { continuous_profile }(d p)= \\
& \left\{(p, s) \mid \exists i:\left(p_{i}, s_{i}\right) \in d p \wedge\right. \\
& \left(p_{i+1}, s_{i+1}\right) \in d p \wedge \\
& \left(\left(p_{i}<p<p_{i+1}\right) \wedge s=s_{i}\right) \\
& \vee \\
& \left(p=p_{i+1} \wedge\right. \\
& \left(s_{i} \leq s_{i+1} \rightarrow s_{i} \leq s \leq s_{i+1}\right) \\
& \wedge \\
& ) \\
& \left(s_{i+1}<s_{i} \rightarrow s_{i+1} \leq s \leq s_{i}\right) \\
& \}
\end{aligned}
$$

\section{Using Profiles to Model Con- straints}

In the context of the relational operators $R=\{\langle\rangle$,$\} ,$ a profile can be used to define a constraint as follows:
Definition 4 Let $P$ denote a continuous profile and let $R$ denote either $<$ or $>$. The set $P_{R}=\left\{\left(p_{i}, s_{i}\right) \mid\right.$ $\left.\forall\left(p_{j}, s_{j}\right) \in P: p_{j}=p_{i} \rightarrow s_{i} R s_{j}\right\}$ is the constraint that results from combining $P$ and $R$.

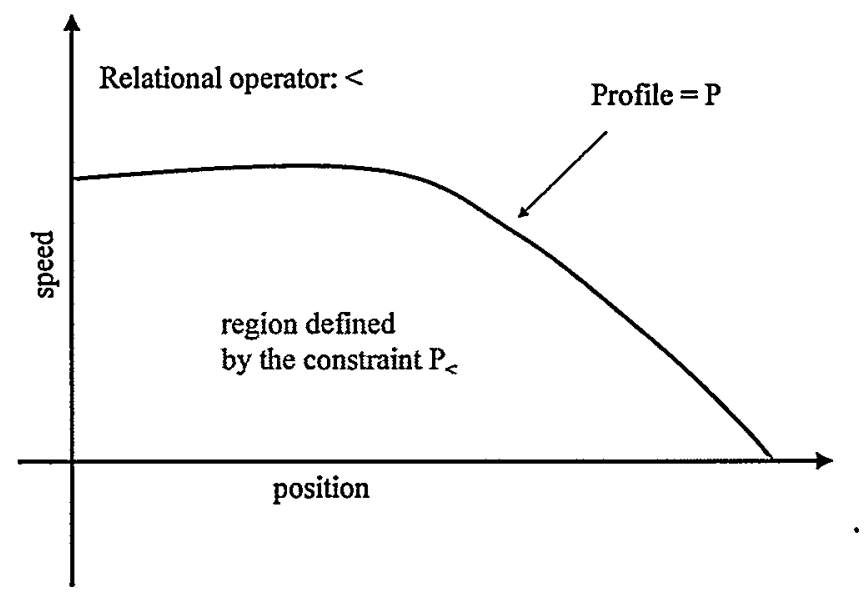

Figure 3: Using a profile to define a constraint

Definition $5 P \ll C_{<} \Leftrightarrow \forall(p, s) \in P:(p, s) \in C_{<} \vee$ $\neg \exists s^{\prime}:\left(p, s^{\prime}\right) \in C_{<}$

When $P \ll C_{<}$holds, we say the profile $P$ satisfies the constraint $C_{<}$.

Definition 6 Let $C_{1}, C_{2}, \ldots, C_{n}$ denote a collection of constraints. The expression $A \ll\left[C_{1}, C_{2}, \ldots, C_{n}\right]=$ $A \ll C_{1} \wedge \ldots \wedge A \ll C_{n}$

Let $\left(m_{1},\left(a_{1}, b_{1}\right)\right)$ denote the current system state at time $t_{i}^{-}$. Let $T$ denote the continuous track profile, and let $L T$ and $O B$ respectively denote the continuous stopping profiles of the lead train and the object train at time $t_{i}^{-}$. Furthermore, suppose that we are given that the object train presently has a normal acceleration (i.e., $a_{1} \in \mathcal{N} \mathcal{A}\left(a_{0}\right)$ where $a_{0}$ is the acceleration value in the previous time step). Under these conditions, the following statement holds:

Theorem 1 If $O B \ll\left[T_{<}, L T_{<}\right]$, then the system is in a normal state (from the perspective of the object train). That is, $\left(m_{1},\left(a_{1}, b_{1}\right)\right) \in \mathcal{N} \mathcal{S}$.

The ideas presented in the theorem above provide the framework for adding additional constraints (such as signals and stations) to a system model. The general approach is to define normal and abnormal states with respect to a collection of constraints (e.g., track 
speed limits, lead train, signals, and stations). After this is done the above theorem can be recast in the appropriate form.

Theorem 2 if $A^{\prime}=\operatorname{continuous}(A)$ and $B^{\prime}=$ continuous $(B)$ then

$$
\begin{aligned}
& A^{\prime} \ll B_{<}^{\prime} \\
& \Leftrightarrow \\
& \left(\forall\left(p_{i}, s_{i}\right),\left(p_{i+1}, s_{i+1}\right) \in A:\right. \\
& \forall\left(p_{j}, s_{j}\right) \in B: \\
& ) \\
& \left(p_{i} \leq p_{j} \leq p_{i+1} \rightarrow\left(s_{i}<s_{j}\right)\right) \\
& \wedge \\
& \left(\forall\left(p_{i}, s_{i}\right) \in A:\right. \\
& \forall\left(p_{j}, s_{j}\right),\left(p_{j+1}, s_{j+1}\right) \in B: \\
& )\left(p_{j} \leq p_{i} \leq p_{j+1} \rightarrow\left(s_{i}<s_{j}\right)\right)
\end{aligned}
$$

\subsection{A Refined View of Safety}

In the informal specification of BART, a worst case stopping profile is defined. The worst case stopping profile is a function that conservatively estimates the longest distance a train could travel, in emergency stop mode, before it comes to a dead stop. Given a worst case stopping profile function, $\mathcal{W}$, we conservatively assume that the distance between any two trains must always be greater-than the distance computed by $\mathcal{W}$. Furthermore, we now can change/extend the definition of abnormal state so that if the distance between two trains is ever equal-to or less-than the emergency stopping profile then, an abnormal state exists, and for safety reasons, the object train must issue the emergency stop command. The reason being that if the lead train were to derail at this point in time, then the object train could collide with it as well, leading to a cascading failure.

It is worth noting that our discussion of basic safety, in Section 3.1.1, does not consider the worst case stopping profile function. This does not pose any significant problems because, given the definition of function for computing the worst case stopping profile, the computed distance can simply be added to the position of the object train. This in effect shifts (and slightly perturbs) the stopping profile of the object train. Another way of viewing the effect of the worst case stopping profile is that it "projects" an image of the object train ahead of itself, and it is this image that controller needs to prevent from colliding with the lead train. Thus, it is the profile of this image that must satisfy the constraints of the system in order for the system to be in a safe state.

\section{The Domain Language}

The domain language we have developed is a functional-style language that provides operators and data structures that are well suited for formally expressing algorithmic specifications of reactive systems in the manner that we have discussed in this paper. Some operators of interest that are supported by the domain language are:

- $\ll \stackrel{\text { signature }}{=}$ profile $\times$ constraint $\rightarrow$ boolean. This operation is used to determine whether a profile satisfies a constraint.

- quantification: $\exists$ and $\forall$

- : $\stackrel{\text { signature }}{=}$ set $\times$ predicate $\rightarrow$ set. The expression $S: P$ produces the subset $S^{\prime}$ of $S$ containing all the elements in $S$ that satisfy $P$.

The domain language also supports a vector and vector sequence data structure that is useful for modeling systems. A useful operation on vectors is the projection of subvectors. In the domain language projections are defined in terms of index lists. For example, given a vector $v=\left(x_{1}, x_{2}, x_{3}, x_{4}\right)$, the expression $v[1,4]$ denotes the subvector $\left(x_{1}, x_{4}\right)$. An operation that is useful on vector sequences is the extraction of subvector sequences. Given a vector sequence $v_{\text {seq }}=$ $\left\langle v_{1}, v_{2}, \ldots, v_{n}\right\rangle$, the expression $v_{s e q}[\mid 1,4]$ denotes the vector subsequence $\left\langle v_{1}[1,4], v_{2}[1,4], \ldots, v_{n}[1,4]\right\rangle$. In the specification of BART, extraction is useful for obtaining profiles.

\subsection{Problem Specific Information}

In addition, each system specification is responsible for defining the following modeling concepts:

- Problem specific definitions:

$-\gamma t-a$ function that changes the control variables but not the monitored variables

- $\Delta t-a$ function that changes the monitored variables but not the control variables

- Stopping profile

- Enumerations of control variable domains

- Set/vector selection mechanisms (e.g., max). 


\subsection{Foundation}

The semantics of the domain language are defined in a standard functional framework (e.g., anonymous functions, recursion, etc.) that has been extended with six key functions: list, constrain, $m u$, list_mu, project, and extract. While there is always the possibility of adding additional functions, we have found that these six functions provide a solid and relatively complete framework enabling efficient implementations to be derived from specifications.

The function list: element $\times$ next_element $\times$ cardinality $\rightarrow$ enumerated_set is used to constructively generate an enumerated set of elements given an initial element, a function for generating the next element, and an integer indicating how many elements are to be generated (e.g., the cardinality of the set).

The function constrain : set $\times$ predicate $\rightarrow$ set has been defined in the previous section, where it is denoted by the colon symbol. The function $m u$ : set $\times$ predicate $\rightarrow$ set is a function that traverses an enumerated set in the order defined by the enumeration and returns an enumerated subset containing the first element, $x$, encountered which satisfies the input predicate as well as all the elements preceding $x$ in enumerated input set. The function list_mu : element $\times$ next_element $\times$ predicate $\rightarrow$ set is a function that generates the set felement, next_element ${ }^{1}$ (element), next_element ${ }^{2}$ (element)... $\}$. In the case where list_mu $(x, n e x t, P)$ is finite, all elements except for the last do not satisfy $P$, and the last element satisfies $P$.

The function project $:$ vector $\times$ index_list $\rightarrow$ vector is used to extract subvectors from a vector, and the function extract : vector_seq $\times$ index_list $\rightarrow$ vector_seq is used for extracting subsequences of vector sequences.

- Let $\vec{v}=\left(v_{1}, v_{2}, v_{3}, v_{4}, v_{5}, v_{6}\right)$ denote a vector. Then $\operatorname{project}(\vec{v},[1,2,5])=\left(v_{1}, v_{2}, v_{5}\right)$.

- Let $v_{s e q}=\left(\left(p_{1}, s_{1}, a_{1}\right),\left(p_{2}, s_{2}, a_{2}\right), \ldots,\left(p_{n}, s_{n}, a_{n}\right)\right)$ denote a vector sequence. Then $\operatorname{extract}\left(v_{\text {seq }}\right.$, $[1,2])=\left(\left(p_{1}, s_{1}\right),\left(p_{2}, s_{2}\right), \ldots,\left(p_{n}, s_{n}\right)\right)$.

Below are the operational definitions of these functions:

\section{- Set Enumeration:}

define list $=($ lambda

$$
(x, \text { next, } 0) \Rightarrow[]
$$$$
\mid(x, \text { next }, n) \Rightarrow x:: \text { list }(\text { next }(x), \text { next }, n-1)
$$

);

- Constraining Sets:

define constrain $=($ lambda set, $P$.

if $n=0$

then set

else if $\mathcal{P}$ (first(set))

then first(set) :: constrain(rest(set), $\mathcal{P})$

)

- Mu operators:

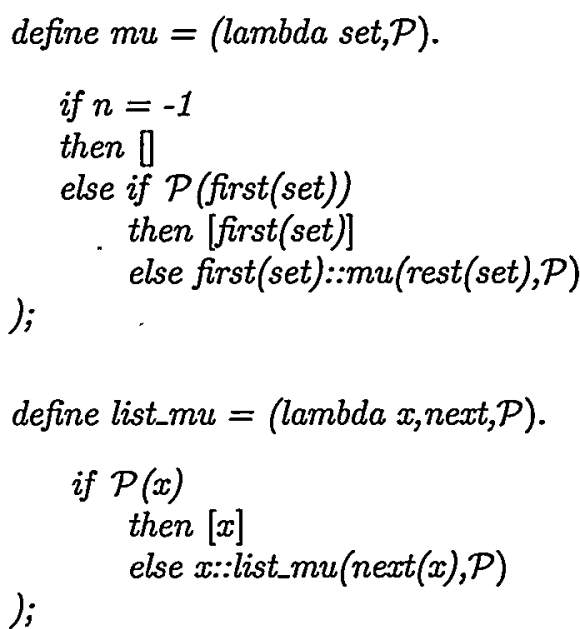

- The projection operator:

define project $=(l a m b d a$

);

$$
(x s,[]) \Rightarrow[]
$$

$\mid(x s, y:: y s)=>n t h(x s, y):: p r o j e c t(x s, y s)$

- The extract:

define extract $=($ lambda

$$
\begin{gathered}
([I, y s)=>I] \\
\mid(x:: x s, y s)=>\operatorname{project}(x, y s):: \operatorname{extract}(x s, y s)
\end{gathered}
$$

The constrain function can be used to define the semantics of universal and existential quantification on enumerated sets as follows: 
- Quantifiers:

- Given a variable $x$, let domain( $x$ ) denote the enumerated set over which $x$ is quantified.

$-\forall x . P(x) \stackrel{\text { def }}{=} \operatorname{domain}(x): P=\operatorname{domain}(x)$

$-\exists x . P(x) \stackrel{\text { def }}{=} \operatorname{domain}(x): P \neq \emptyset$

\section{The Specification of BART}

At this point a domain language has been developed in which controllers for BART can be specified. For example, a controller for the object train of a two train system can be specified in the following manner:

- Define the following set of vectors of interest:

- A vector describing the system state as described in Section 2. In this example, the state of the system can be modeled by $s=\left(p_{1}, s_{1}, p_{2}, s_{2}, t, a_{1}, a_{2}\right)$, where $\left(p_{1}, s_{1}, p_{2}, s_{2}, t\right)$ denotes the vector of monitored variables and $\left(a_{1}, a_{2}\right)$ denotes the vector of controlled variables.

- A vector describing the state of a train. Given the state of the system, $s$, the state of the object train is described by the projection $s[f 1]=s[1,2,6]=\left(p_{1}, s_{1}, a_{1}\right)$, and the lead train is described by the projection $s[f 2]=s[3,4,7]=\left(p_{2}, s_{2}, a_{2}\right)$.

- A vector describing the profile elements of a trace (i.e., its position and speed variables). Given the state of the system, $s$, the track profile is described by the projection $s[f 3]=$ $s[5]=(t)$

- Define functions capable of enumerating the set of acceleration values according to the physical limitations of the system.

- Define the constraints on the controller. In this case, the controller for the object train should not violate the constraints of the lead train's stopping profile and the track profile.

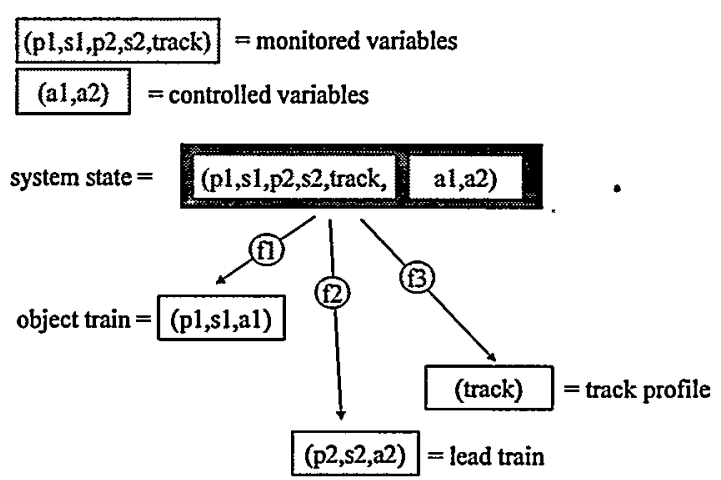

Figure 4: Relationship of Various Vectors to the System State Vector

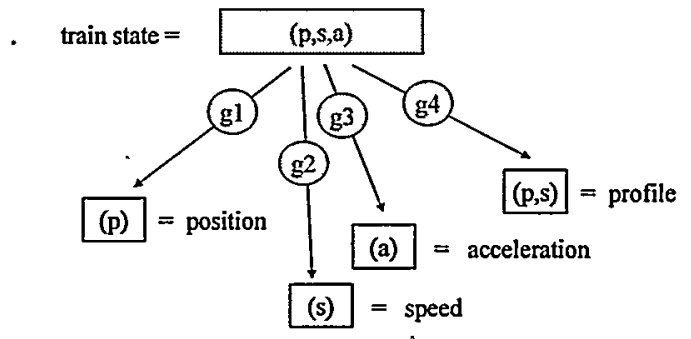

Figure 5: Relationship of Various Vectors to the Train State Vector

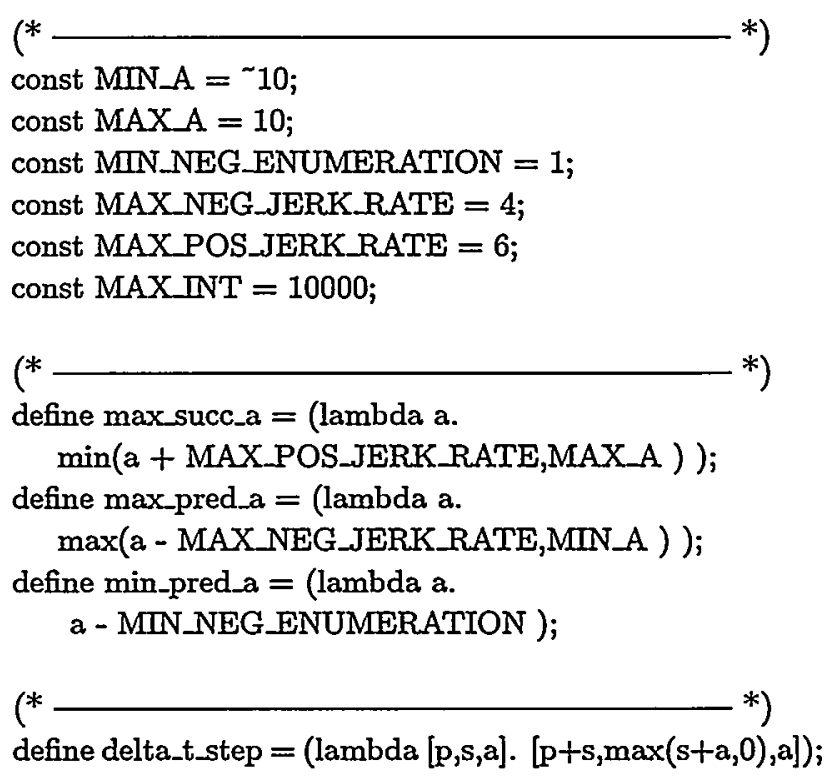




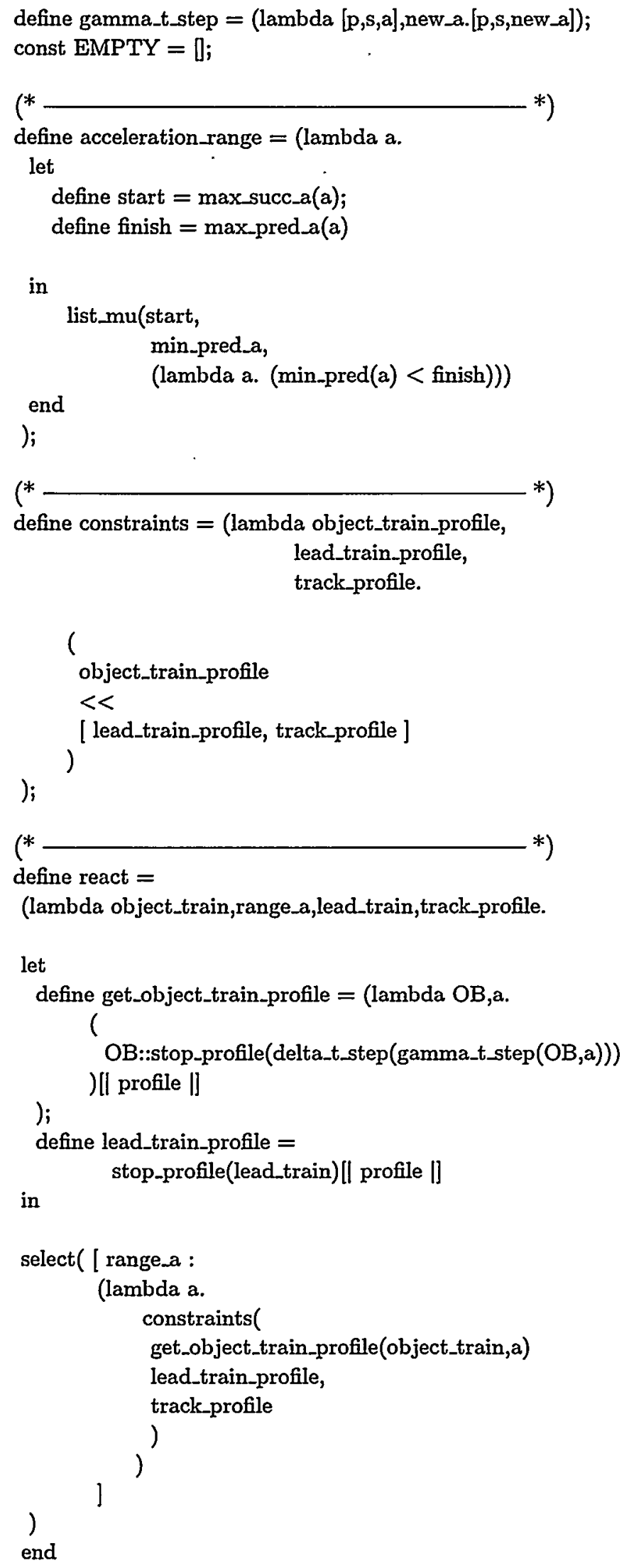

)

(*

define controller $=$

(lambda system_state.

let

define object_train_state $=$ system_state[fl]

define lead_train_state $=$ system.state[f2];

define track_profile $=$ system_state[f3]

in

react(object_train_state, acceleration_range(object_train_state[g3]), lead_train_state, track_profile)

end

);

(*

Notable aspects of BART that are simplified or omitted from this specification are:

- The actual model of acceleration. In BART, a change from positive to negative acceleration (and vice versa) must be preceded by a mode change. Furthermore, the maximum acceleration is dependent upon the speed the train is traveling. This acceleration function can easily be specified as a large case statement defined on an enumerated set having "mode change" elements at the appropriate positions.

- The position and speed computations have been simplified in our specification.

- Our specification does not consider the impact of noisy/lossy transmissions (e.g., the object train gets a garbled acceleration command). In our specification, noise can be accounted for by properly defining how it impacts the stopping profile of the object train. The rest of the specification then remains unchanged.

The specification given above can be easily altered to include more appropriate models (as defined by domain experts). The objective of this research is not to demonstrate our domain knowledge, but rather to address the issues surrounding the specification of a class of reactive system controllers of which BART is an instance. 


\section{Conclusions and Future Work}

We believe that domain languages that are well suited for the formal specification, design, and implementation of problems have the following characteristics: (1) the problem domain is sufficiently restricted so that a small set of operations and data structures can be used to model and specify the problem, (2) the operations and data structures provide a framework for understanding nondeterminism, and (3) a simple operational semantics exists for the operators and data structures of the domain language.

The domain language described in this paper was restricted to reactive systems having train-like characteristics. We discovered that profiles, both continuous and discrete, and their extensions to constraints were key to understanding and specifying systems. Furthermore, we found that a vector datatype supporting the operations project and extract was fundamental to model these systems. And lastly, it was through $\gamma t$ and $\Delta t$ transitions that time could be understood with respect to our system model.

We are currently working on extending the domain language to enable the specification of multi train systems and are also considering how other environmental factors, such as noisy transmission of control information, can be accounted for in a specification. Another interesting problem is how the control of a train can be handed off from one controller to another.

\section{References}

[1] K. A. Ghosh, B. W. Johnson, and J. A. Profeta, III. A Distributed Safety-Critical System for RealTime Train Control. Proceedings of the $21^{\text {st }}$ Annual Conference of the IEEE Industrial Electronics Society (IECON '95), Orlando, Florida, November 6-10, 1995, pp. 760-767.

[2] H. Gomaa. Software Design Methods for Concurrent and Real-Time Systems. Addison Wesley, 1993.

[3] B. Johnson and J. H. Aylor. Reliability and Safey Analysis of a Fault-Tolerant Controller. IEEE Transactions on Reliability, Vol. R-35, No. 4, October 1986, pp. 355-362.

[4] C. Lewerentz and T. Lindner. Formal Development of Reactive Systems: Case Study Production Cell. Lecture Notes in Computer Science Vol. 891, Springer-Verlag.
[5] Z. Manna and A. Pnueli. Temporal Verification of Reactive Systems: Safety. Springer-Verlag, 1995.

[6] R. Janicki, D. Parnas, and J. Zucker. Tabular Representations in Relational Documents. CRL Report No. 313, November 1995.

[7] Victor Winter, Raymond Berg, and Jim Ringland. Bay Area Rapid Transit District Advance Automated Train Control System Case Study Description. Proceedings of the 1999 High Integrity Software (HIS) Conference. 